Supporting information to:

\title{
Raman activity of multilayer phosphorene under strain
}

\author{
K. Tokár ${ }^{1}$, J. Brndiar ${ }^{1}$, I. Štich ${ }^{1,2, *}$ \\ ${ }^{1}$ Center for Comp. Mat. Science, Inst. of Physics, Slovak Acad. of Sciences, \\ 84511 Bratislava, Slovakia \\ ${ }^{2}$ Inst. of Informatics, Slovak Acad. of Sciences, 845 07, Bratislava, Slovakia
}

*Electronic address: ivan.stich@savba.sk 


\section{Structure of the strained system}

Structural parameters of unstrained system are shown in Fig. S1 and their DFT optimized values given in Tab. S I.

Evolution of structural parameters under applied armchair and zigzag strain are shown in Fig. S2 and Fig. S3, respectively. These results show that the interlayer spacings $h_{i}$ increase under applied armchair tensile strain, Fig. S2, thus representing a theoretical confirmation of negative Poisson's ratio in FLP experimentally deduced from low-frequency breathing modes [1]. Interestingly, this behavior is limited to armchair strain and no such behavior is found when the strain is applied in the zigzag direction, Fig. S3. Similar behavior was found also previously on a three-layer phosphorene [2].
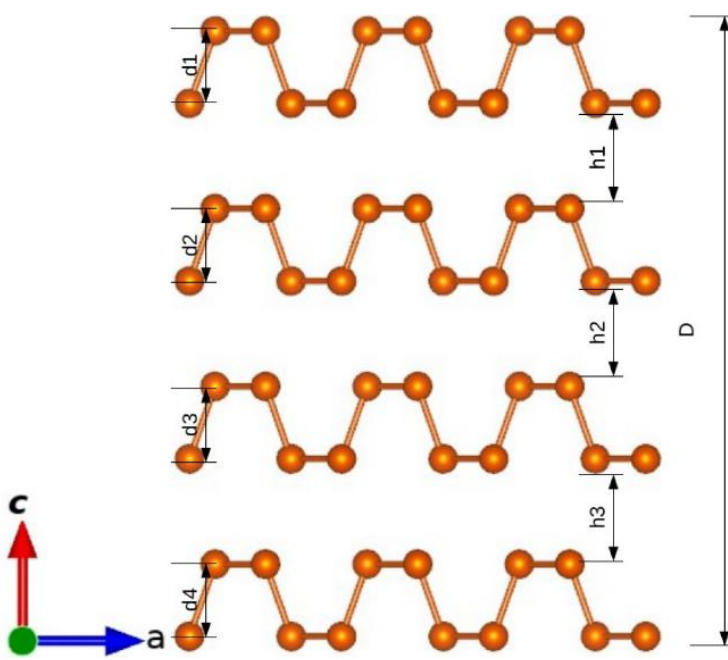

Supporting Figure S1. Side view of atomic structure of a four-layer BP. The principal structural parameters $d_{i}, h_{j}$, and $D$ are shown.

Supporting Table S I. Four-layer structure parameters of an unstrained structure: layer thickness $d_{i}$, distance between the layers $h_{j}$ and total FLP thickness $D\left(d_{1}=d_{4}, d_{2}=d_{3}, h_{1}=h_{3}\right.$ by symmetry) are given. All parameters in $\AA$. Lattice parameters: $a=3.315 \AA, b=4.474 \AA, D=$ $17.834 \AA$.

\begin{tabular}{|c|c|c|c|c|c|c|c|}
\hline $\boldsymbol{d}_{\boldsymbol{1}}$ & $\boldsymbol{d}_{\boldsymbol{2}}$ & $\boldsymbol{d}_{\mathbf{3}}$ & $\boldsymbol{d}_{\boldsymbol{4}}$ & $\boldsymbol{h}_{\boldsymbol{1}}$ & $\boldsymbol{h}_{\boldsymbol{2}}$ & $\boldsymbol{h}_{\mathbf{3}}$ & $\boldsymbol{D}$ \\
\hline 2.139 & 2.145 & 2.145 & 2.139 & 3.087 & 3.089 & 3.087 & 17.834 \\
\hline
\end{tabular}

Evolution of structural parameters under applied armchair and zigzag strain are shown in Fig. S2 and Fig. S3, respectively. These results show that the interlayer spacings $h_{i}$ increase under applied armchair compressive strain, Fig. S2, thus representing a theoretical confirmation of negative Poisson's ratio in FLP experimentally deduced from low-frequency breathing modes [1]. Interestingly, this behavior is limited to armchair strain and no such behavior is found when the strain is applied in the zigzag direction, Fig. S3. Similar behavior was found also previously on a three-layer phosphorene [2].

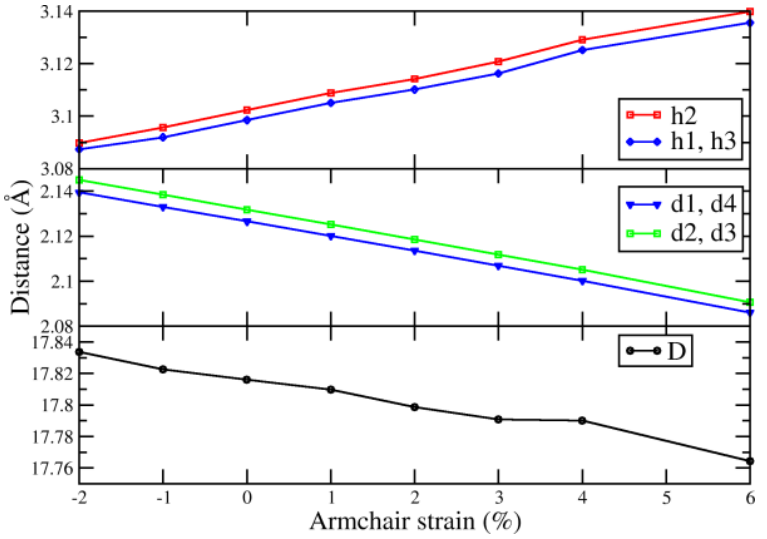

Supporting Figure S2. Evolution of key structural parameters in a four-layer under strain applied in armchair direction. 


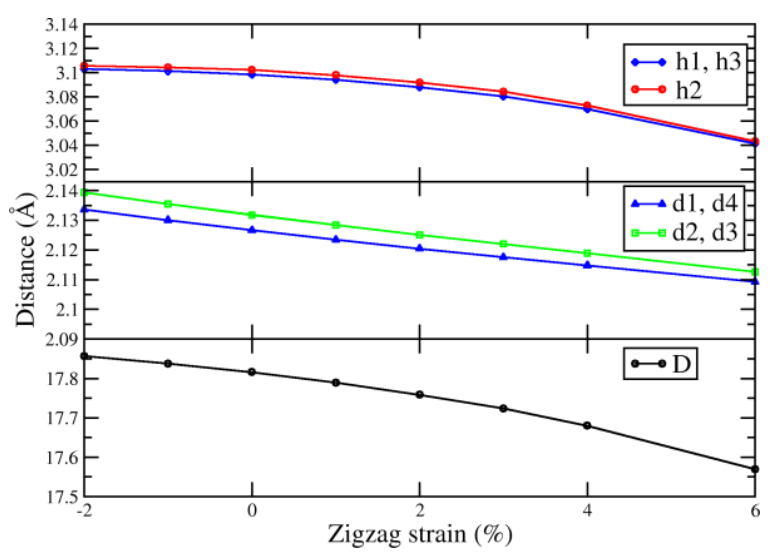

Supporting Figure S3. Evolution of key structural parameters in a four-layer under strain applied in zigzag direction.

\section{Dominant Raman modes in unstrained FLP}

The computed (PW) and experimentally measured $[3,4]$ frequencies of the main high-frequency Raman modes are compiled for comparison in Tab. S II. As general rule, the frequency of the $A_{g}{ }^{2}$ mode decreases with the number of layers $L$, whereas the frequencies of the other two modes, $B_{2 g}$ and $A_{g}{ }^{l}$ are barely affected. Some experimental scatter between the experimental sets of a few $\mathrm{cm}^{-1}$ can be seen. The computed frequencies are all shifted to lower frequencies by $\approx 15 \mathrm{~cm}^{-1}$. We believe this shift is due to a combination of absence of the dielectric embedding in the model and possible computational biases, such as basis set cut-offs, use of pseudopotentials, etc.

The angular dependence of activities of the HF $\mathrm{R}$ modes is shown in Fig. S4. The activity of the $A g$ modes changes with the variation period of $180^{\circ}$, while variation period of the $B_{2 g}$ is $90^{\circ}$. Note that activity of the $B_{2 g}$ mode is significantly

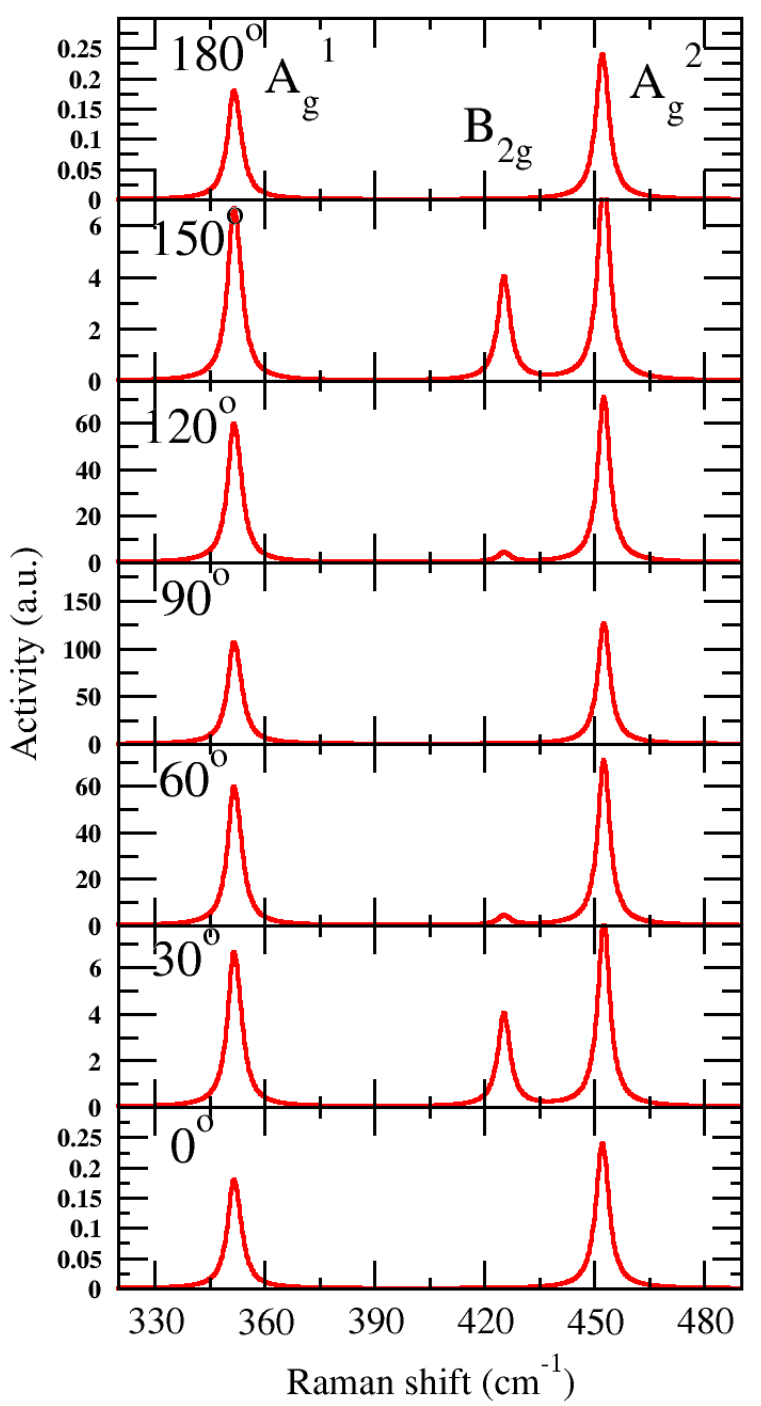

Supporting Figure S4. Angular dependence of activities of HF R modes in a four-layer phosphorene. Note the different vertical scale for the different angles.

smaller than that of the $A$ modes but is large at angles where also activity of the $A$ modes is small making the intensities of all three modes comparable at angles around $30^{\circ}$. 
Supporting Table S II. Comparison of the main high-frequency Raman modes, $A_{g}{ }^{2}, B_{2 g}$, and $A_{g}{ }^{l}$, experimentally measured $[3,4]$ and from the present modeling (PW).

\begin{tabular}{l|c|c|c|c|c|c|c|c|c|c|c|c}
\hline mode/\#layers & \multicolumn{3}{|c|}{ L1 } & \multicolumn{3}{c|}{ L2 } & \multicolumn{3}{c|}{ L3 } & \multicolumn{3}{c}{ L4 } \\
\hline Ref. & {$[3]$} & {$[4]$} & PW & {$[3]$} & {$[4]$} & PW & {$[3]$} & {$[4]$} & PW & {$[3]$} & {$[4]$} & PW \\
\hline $\boldsymbol{A}^{\boldsymbol{g}} \boldsymbol{g}$ & 471.5 & 467.6 & 456.1 & 470.0 & 467.0 & 454.4 & 469.2 & 466.0 & 451.6 & 468.8 & 465.0 & 452.6 \\
\hline $\boldsymbol{B}_{\boldsymbol{2} \boldsymbol{g}}$ & 440.2 & 439.3 & 422.8 & 440.7 & 438.6 & 423.3 & 440.3 & 438.0 & 419.8 & 440.2 & 437.2 & 422.9 \\
\hline $\boldsymbol{A}^{\boldsymbol{I}} \boldsymbol{l}$ & - & 362.5 & 353.0 & - & 362.0 & 352.0 & - & 361.5 & 352.5 & - & 361.0 & 351.5 \\
\hline
\end{tabular}

Influence of the inter-layer electronic coupling by comparing selected vibrational frequencies in the presence and absence of the D2 correction [5] to PBE is shown in Tab. SIII.

Supporting Table S III. Comparison of the main Raman modes, $A_{g}{ }^{2}, B_{2 g}, A_{g}{ }^{1}, B 1$, and $B 2$ in DFT-D2 (vdW) and in pure PBE (no vdW) treatment.

\begin{tabular}{l|c|c|c|c|c|c|c|c}
\hline \multirow{2}{*}{ mode/\#layers } & \multicolumn{2}{|c|}{ L1 } & \multicolumn{2}{c|}{ L2 } & \multicolumn{2}{c}{ L3 } & \multicolumn{2}{c}{ L4 } \\
\cline { 2 - 10 } & vdW & no vdW & vdW & no vdW & vdW & no vdW & vdW & no vdW \\
\hline $\boldsymbol{A}^{\mathbf{2} g}$ & 456.1 & 451.9 & 454.4 & 450.3 & 451.6 & 447.87 & 452.6 & 449.0 \\
\hline $\boldsymbol{B}_{\boldsymbol{2} \boldsymbol{g}}$ & 422.8 & 422.3 & 423.3 & 422.1 & 419.8 & 417.0 & 422.9 & 418.9 \\
\hline $\boldsymbol{A}^{\boldsymbol{I} g}$ & 353.0 & 347.9 & 352 & 347.6 & 352.5 & 347.7 & 351.5 & 344.9 \\
\hline B2 & & & 69.6 & 58.6 & 49.9 & 42.0 & 91.6 & 76.0 \\
\hline B1 & & & & & & & 38.3 & 32.5 \\
\hline
\end{tabular}

\section{Mode decomposition at the $\Gamma$-point of the Brillouin zone}

Here we provide vibrational mode decomposition at the $\Gamma$-point of the Brillouin zone for the four-layer phosphorene structure, space group Pmca (\#57):

$8 A_{g}+4 B_{1 g}+4 B_{2 g}+8 B_{3 g}+7 B_{1 u}+7 B_{2 u}+3 B_{3 u}+$ $4 \mathrm{~A}_{\mathrm{u}}$

Raman active: $A_{g}, B_{2 g}, B_{1 g}, B_{3 g}$

IR active: $\quad B_{1 u}, B_{2 u}, B_{3 u}$

Silent: $\quad A_{u}$ 
Supplorting Table S IV: Raman tensors of selected modes in four-layer phosphorene.

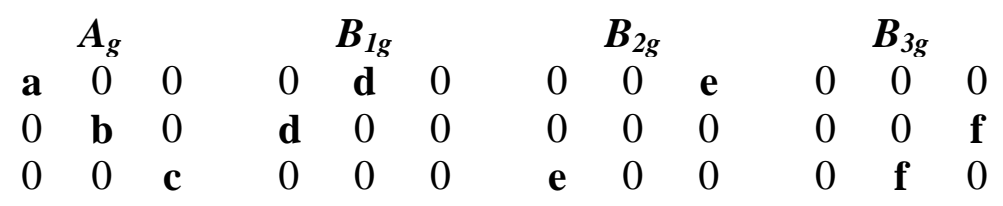

\section{Raman activities in FLP under armchair and zizgzag strain}

In Fig. S5 we show the frequency shifts of the activity of the high-frequency Raman modes under armchair and zigzag strain for a single-layer, tri-layer, and four-layer phosphorene. This figure is complementary to Fig. 3 of the main text and shows the trend robustness.
Evolution of Raman activity of dominant modes in four-layer phosphorene on number of layers and on armchair strain is shown on a logarithmic scale in Fig. S6. This serves to visualise the much weaker $B_{2 g}$ mode.
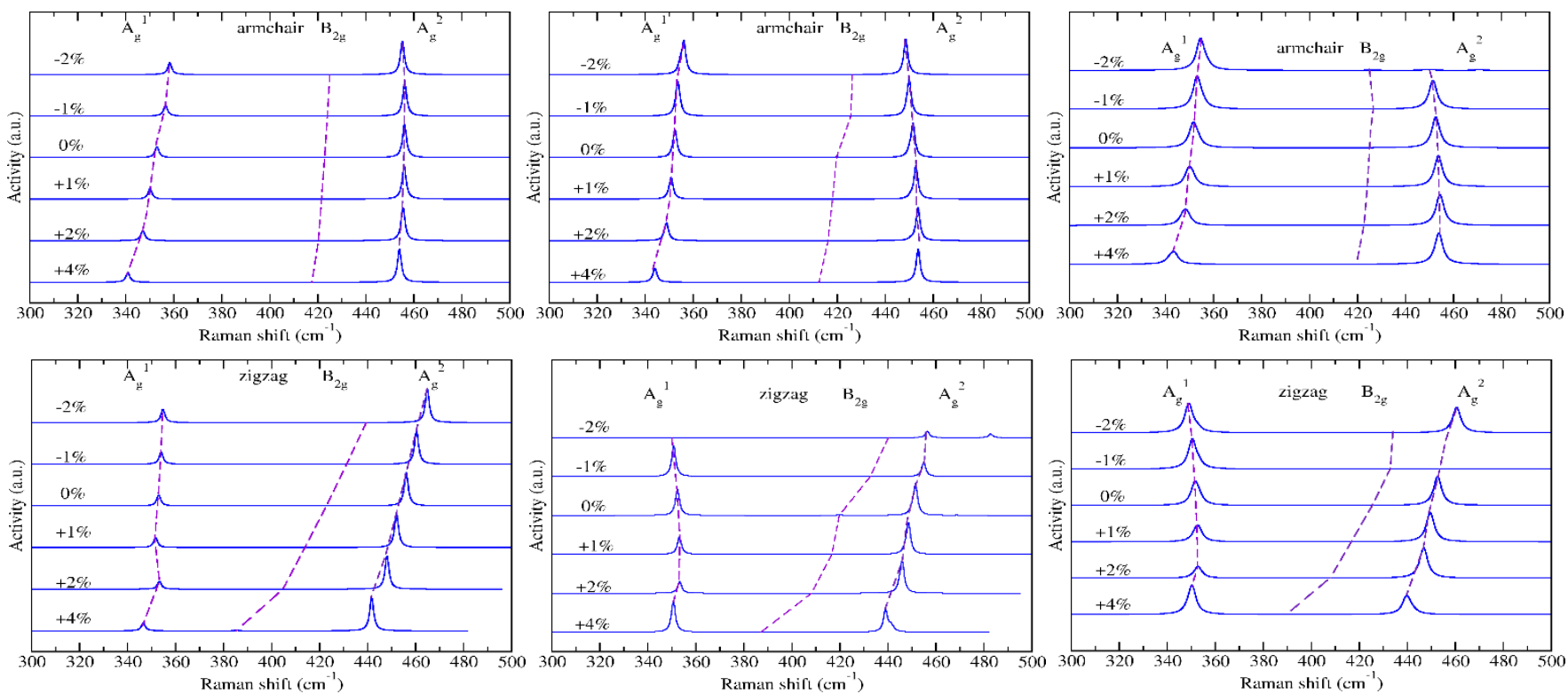

Supporting Figure S5. Evolution of the key high-frequency Raman modes in single-layer (left panels), tri-layer (middle panels), and four-layer (right panels) phosphorene under strain applied in armchair (top) and zigzag (bottom) direction. 

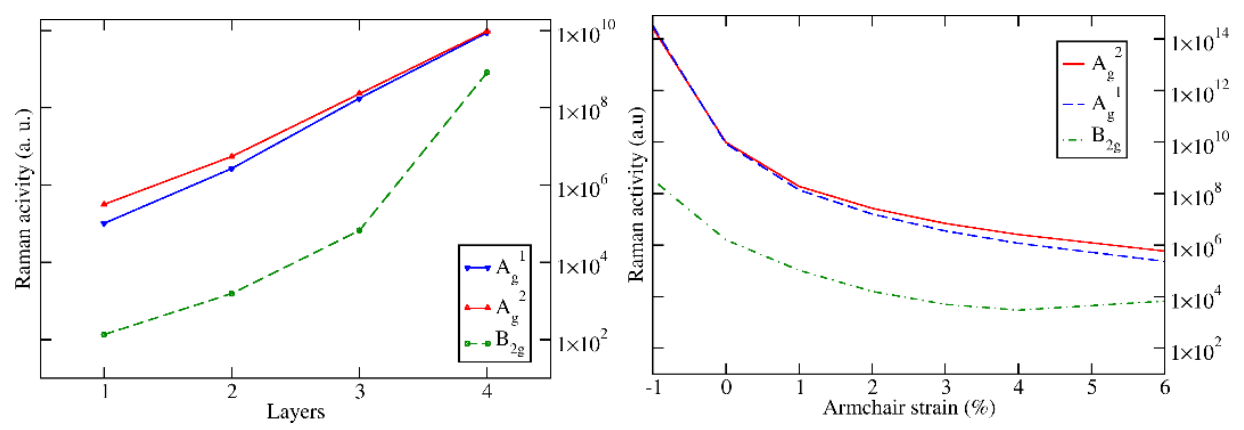

Supporting Figure S6. Evolution of Raman activity of dominant high-frequency modes in fourlayer phosphorene on number of layers (left) and on armchair strain (right) on a logarithmic scale.

\section{Evolution of DFT HOMO-LUMO gaps under applied strain}

As example in Tab. S V we show evolution of DFT HOMO-LUMO gap under applied armchair and zigzag strain. Note the small values of the gap due to layer thickness and gap underestimation of the quasiparticle gaps in customary DFT description compared to correlated treatments [6]. The corresponding electronic gaps are shown in Fig. S7.

Supporting Table $\mathbf{S ~ V}$. Evolution of DFT HOMO-LUMO gaps under applied armchair and zigzag strain in four-layer phosphorene.

\begin{tabular}{c|c|c|c|c}
\hline \multirow{2}{*}{$\begin{array}{c}\text { strain } \\
(\boldsymbol{\%})\end{array}$} & \multicolumn{2}{|c|}{$\mathbf{A C}$} & \multicolumn{2}{c}{$\mathbf{Z Z}$} \\
\cline { 2 - 5 } $\boldsymbol{\Gamma} \rightarrow \boldsymbol{\Gamma}$ & $\Gamma \rightarrow \mathbf{Y}$ & $\Gamma \rightarrow \Gamma$ & $\Gamma \rightarrow \mathbf{Y}$ \\
\hline-4 & 0.00 & 0.00 & 0.00 & 0.00 \\
-2 & 0.00 & 0.00 & 0.05 & 0.00 \\
-1 & 0.00 & 0.00 & 0.00 & 0.00 \\
0 & 0.08 & 0.08 & 0.08 & 0.08 \\
+1 & 0.16 & 0.12 & 0.16 & 0.14 \\
+2 & 0.23 & 0.14 & 0.26 & 0.19 \\
+3 & 0.30 & 0.16 & 0.36 & 0.24 \\
+4 & 0.37 & 0.19 & 0.35 & 0.30 \\
+6 & 0.47 & 0.25 & 0.36 & 0.33 \\
\hline
\end{tabular}
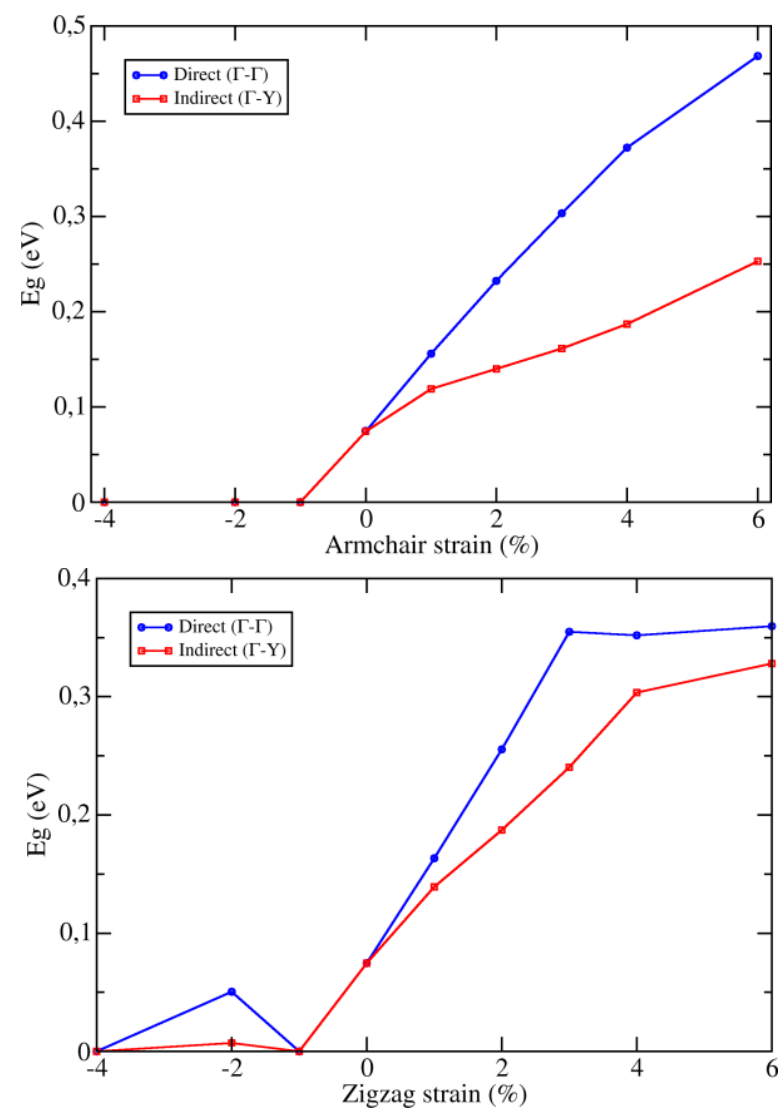

Supporting Figure S7. Evolution of the DFT HOMO-LUMO gap in a four-layer structure under strain applied in armchair (top) and zigzag (bottom) direction 
Evolution of the corresponding electronic band structure under applied armchair strain is shown in Fig. S8. One can see that at negative strain a cone develops in the $\Gamma \rightarrow$ $\mathrm{Y}$ direction and that at positive strain a minimum of the conduction band shifts from $\Gamma$ towards the $\mathrm{Y}$ point.

Supporting Figure S8. Evolution of the DFT electronic structure in a four-layer structure under strain applied in armchair direction. From top to the bottom: $-4 \%,-3 \%$, $-2 \%, 0.0 \%$, and $+4.0 \%$
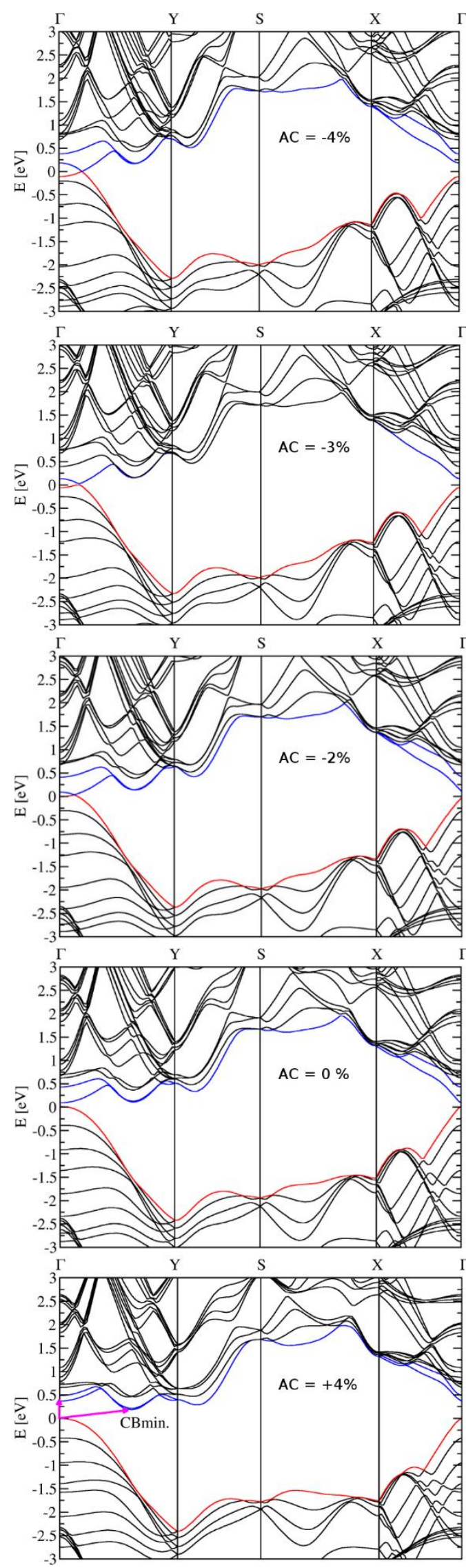
[1] Y. Du, J. Maassen, Z. Wu, W. Luo, X. $\mathrm{Xu}$, and P. D. Ye, Nano Lett. 16, 6701 (2016).

[2] K. Tokár, R. Derian, J. Brndiar, and I. Štich, J. Appl. Phys. 120, 194305 (2016).

[3] W. Lu, H. Nan, J. Hong, Y. Chen, C. Zhu, Z. Liang, X. Ma, Z. Ni, C. Jin, and Z. Zhang, Nano Res. 7, 853 (2014).

[4] Z. Guo, H. Zhang, S. Lu, Z. Wang, S. Tang, J. Shao, and P. K. Chu, Adv. Funct. Mater. 25, 7100 (2015).

[5] S. Grimme, J. Comp. Chem. 27, 1787 (20016).

[6] T. Frank, R. Derian, K. Tokár, L. Mitas, J. Fabian, and I. Štich, Phys. Rev. X 9, 011018 (2019). 